\title{
ML-Leaks: Model and Data Independent Membership Inference Attacks and Defenses on Machine Learning Models
}

\author{
Ahmed Salem*, Yang Zhang*§, Mathias Humbert ${ }^{\dagger}$, Pascal Berrang*, \\ Mario Fritz*, Michael Backes* \\ ${ }^{*}$ CISPA Helmholtz Center for Information Security, \\ \{ahmed.salem, yang.zhang, pascal.berrang, fritz, backes\}@cispa.saarland \\ ${ }^{\dagger}$ Swiss Data Science Center, ETH Zurich and EPFL, mathias.humbert@epfl.ch
}

\begin{abstract}
Machine learning (ML) has become a core component of many real-world applications and training data is a key factor that drives current progress. This huge success has led Internet companies to deploy machine learning as a service (MLaaS). Recently, the first membership inference attack has shown that extraction of information on the training set is possible in such MLaaS settings, which has severe security and privacy implications.
\end{abstract}

However, the early demonstrations of the feasibility of such attacks have many assumptions on the adversary, such as using multiple so-called shadow models, knowledge of the target model structure, and having a dataset from the same distribution as the target model's training data. We relax all these key assumptions, thereby showing that such attacks are very broadly applicable at low cost and thereby pose a more severe risk than previously thought. We present the most comprehensive study so far on this emerging and developing threat using eight diverse datasets which show the viability of the proposed attacks across domains.

In addition, we propose the first effective defense mechanisms against such broader class of membership inference attacks that maintain a high level of utility of the ML model.

\section{INTRODUCTION}

Machine learning (ML) has become a core component of many real-world applications, ranging from image classification to speech recognition. The success of ML has recently driven leading Internet companies, such as Google and Amazon, to deploy machine learning as a service (MLaaS). Under such services, a user uploads her own dataset to a server and the server returns a trained ML model to the user, typically as a black-box API.

Despite being popular, ML models are vulnerable to various security and privacy attacks, such as model inversion [12], adversarial examples [15], and model extraction [43], [30], [45]. In this paper, we concentrate on one such attack, namely membership inference attack. In this setting, an adversary aims

$\S$ Corresponding author

Network and Distributed Systems Security (NDSS) Symposium 2019 24-27 February 2019, San Diego, CA, USA

ISBN 1-891562-55-X

https://dx.doi.org/10.14722/ndss.2019.23119

www.ndss-symposium.org to determine whether a data item (also referred to as a data point) was used to train an ML model or not. Successful membership inference attacks can cause severe consequences. For instance, if a machine learning model is trained on the data collected from people with a certain disease, by knowing that a victim's data belong to the training data of the model, the attacker can immediately learn this victim's health status. Previously, membership inference has been successfully conducted in many other domains, such as biomedical data [2] and mobility data [35].

Shokri et al. [38] present the first membership inference attack against machine learning models. The general idea behind this attack is to use multiple machine learning models (one for each prediction class), referred to as attack models, to make membership inference over the target model's output, i.e., posterior probabilities. Given that the target model is a black-box API, Shokri et al. propose to construct multiple shadow models to mimic the target model's behavior and derive the data necessary, i.e., the posteriors and the ground truth membership, to train attack models.

There are two main assumptions made by Shokri et al. [38]. First, the attacker needs to establish multiple shadow models with each one sharing the same structure as the target model. This is achieved by using the same MLaaS that trains the target model to build the shadow models. Second, the dataset used to train shadow models comes from the same distribution as the target model's training data, this assumption holds for most of the attack's evaluation [38]. The authors further propose synthetic data generation to relax this assumption. However, this approach can only be applied to datasets containing binary features for efficiency reasons.

These two assumptions are rather strong which largely reduce the scope of membership inference attacks against ML models. In this paper, we gradually relax these assumptions in order to show that far more broadly applicable attack scenarios are possible. Our investigation shows that indeed, membership inference in ML can be performed in an easier way with fewer assumptions than previously considered. To remedy this situation, we further propose two effective defense mechanisms.

Membership Inference Attack. We study three different types of adversaries based on the design and training data of shadow models. As Table I illustrates, we hereby gradually relax the 


\begin{tabular}{lcccc}
\hline \multirow{2}{*}{ Adversary type } & \multicolumn{2}{c}{ Shadow model design } & \multicolumn{2}{c}{$\begin{array}{c}\text { Target model's } \\
\end{array}$} \\
\cline { 2 - 3 } & No. shadow models & Target model structure & training data distribution \\
\hline Shokri et al. [38] & multiple & $\checkmark$ & $\checkmark$ \\
Our adversary 1 & 1 & - & $\checkmark$ \\
Our adversary 2 & 1 & - & - \\
Our adversary 3 & - & - & - \\
\hline
\end{tabular}

TABLE I: An overview of the different types of adversaries. $\checkmark$ means the adversary needs the information while - indicates the information is not necessary.

assumptions of the previous work until we arrive at model and data independent adversary.

Adversary 1. For the first adversary, we assume she has a dataset that comes from the same distribution as the target model's training data. Here, we concentrate on relaxing the assumptions on the shadow models.

We start by using only one instead of multiple shadow models to mimic the target model's behavior. As shadow models are established through MLaaS, which implements the pay-per-query business model, using one shadow model notably reduces the cost of performing the membership inference attack.

Extensive experimental evaluation (we use a suite of eight different datasets ranging from image to text under multiple types of machine learning models) shows that with one shadow model and one attack model, the adversary can achieve a very similar performance as reported by Shokri et al. [38]. For instance, when the target model is a convolutional neural network (CNN) trained on the CIFAR-100 dataset, ${ }^{1}$ our simplified attack achieves a 0.95 precision and 0.95 recall while the attack with 10 shadow models and 100 attack models (as in the previous work [38]) has a 0.95 precision and 0.94 recall.

Then, we relax the assumption that the shadow model is constructed in the same way as the target model, In particular, we show that training the shadow model with different architectures and parameters still yields comparable attack performance. Moreover, we propose a new approach for shadow model training, which frees the adversary from even knowing the type of ML models used by the target model.

Adversary 2. For this adversary, we assume she does not have data coming from the same distribution as the target model's training data. Also, the adversary does not know the structure of the target model. This is a more realistic attack scenario compared to the previous one.

We propose a data transferring attack for membership inference in this setting. Concretely, we train our single shadow model with a different dataset. This means the shadow model here is not used to mimic the target model's behavior but only to capture the membership status of data points in a machine learning training set.

The main advantage of our data transferring attack is that the adversary does not need to query the target model for synthetic data generation. In contrast, the previous approach [38] requires 156 queries on average to generate a single data point. This means our data transferring attack is much more efficient, less costly, and harder to be detected by the MLaaS provider.

\footnotetext{
${ }^{1}$ https://www.cs.toronto.edu/ kriz/cifar.html
}

Experimental results show that the membership inference attack still achieves a strong performance, with only a few percentage drop compared to the first adversary. More interestingly, we show that our data transferring attack even works between datasets belonging to totally different domains. For example, by training a shadow model with the 20 Newsgroups text dataset, ${ }^{2}$ we are able to get a 0.94 precision and 0.93 recall for attacking a target model trained on the CIFAR-100 image dataset.

Adversary 3. This adversary works without any shadow model, i.e., the attack only relies on the posteriors (outcomes) obtained from the target model when querying it with target data points. No training procedure is required at all. We show that statistical measures, such as maximum and entropy, over the target model's posteriors can very well differentiate member and non-member data points. To make a concrete membership inference, we propose a threshold-choosing method. Experiments show that such a simple attack can still achieve effective inference over multiple datasets.

All these experimental results show that membership inference can be performed in a much simpler and more efficient way, which further demonstrates the severe risks of $\mathrm{ML}$ models.

Defense. To mitigate the membership risks, we propose two defense mechanisms, i.e., dropout and model stacking.

Dropout. One reason behind membership inference attacks' effectiveness is the inherent overfitting nature of machine learning models. When an ML model faces a data point that it was trained on, it returns a high posterior for one class compared to others. Therefore, to defend against membership inference, we use a classical approach adopted in deep learning, namely dropout, which aims at preventing overfitting. Dropout randomly deletes in each training iteration a fixed proportion of edges in a fully connected neural network model.

Experiments on multiple datasets show that dropout can be a very effective countermeasure against membership inference. On the CIFAR-100 dataset, dropout (with 0.5 dropout ratio) decreases the performance of our first adversary from 0.95 precision and 0.95 recall to 0.61 and 0.60 , respectively. Moreover, it almost preserves the same utility as the initial target model: The target model's prediction accuracy only drops from 0.22 to 0.21 (CIFAR-100). As dropout serves as a regularizer, we observe that, for several learning problems, e.g., the Purchase100 dataset [38], the target model's accuracy even improves after applying dropout. Therefore, these models improve in performance and resilience in membership inference attacks.

\footnotetext{
${ }^{2}$ http://scikit-learn.org/stable/datasets/twenty_newsgroups.html
} 
Model Stacking. Although the dropout mechanism is effective, it is specific to deep neural networks. For target models using other machine learning classifiers, we propose a second defense mechanism, namely model stacking. Model stacking is a major class of ensemble learning. In model stacking, multiple ML models are organized in a hierarchical way to prevent overfitting. In our case, we construct the target model with three different machine learning models. Two models are placed in the first layer directly taking the original training data as input, while the third model is trained with the posteriors of the first two models.

Through extensive experiments, we show that model stacking is able to significantly reduce the membership inference's performance. For instance, both precision and recall of the attack (adversary 1) drop by more than $30 \%$ on the CIFAR100 dataset trained with model stacking. Meanwhile, the target model's prediction performance stays almost the same.

In summary, we make the following contributions:

- We broaden the class of membership inference attacks by substantially relaxing the adversarial assumptions.

- We evaluate membership privacy threat under three different adversarial setups on eight diverse datasets, ultimately arriving at a model and data independent adversary. Extensive experiments demonstrate the severe membership privacy threat for machine learning models.

- We propose two defense mechanisms, namely dropout and model stacking, and demonstrate their effectiveness experimentally.

Organization. The rest of the paper is organized as the following. Section II introduces the definition of membership inference against ML models and datasets used in the paper. Section III, Section IV and Section V present the threat models, attack methodologies, and evaluations of our three different types of adversaries, respectively. In Section VI, we introduce the two defense mechanisms. Section VII discusses the related work and Section VIII concludes the paper.

\section{PRELIMINARIES}

In this section, we first define membership inference attack in the machine learning setting. Then, we introduce the datasets used for our evaluation.

\section{A. Membership Inference Against Machine Learning Models}

In this paper, we concentrate on machine learning classification, as it is the most common ML application. An ML classifier is essentially a function $\mathcal{M}$ that maps a data point $\mathcal{X}$ (a multidimensional feature vector) to an output vector $\mathcal{M}(\mathcal{X})=\mathcal{Y}$. The length of $\mathcal{Y}$ is equal to the number of classes considered. For most of the classification models, the output vector $\mathcal{Y}$ can be interpreted as a set of posterior probabilities over all classes, and the sum of all the values in $\mathcal{Y}$ is 1 . The parameters of an ML model are learned on a training dataset (denoted by $\mathcal{D}_{\text {Train }}$ ) containing multiple data points following a predefined learning object.

Membership inference attack in the ML setting emerges when an adversary aims to find out whether her target data point is used to train a certain ML model. More formally, given a target data point $\mathbf{x}_{\text {Target }}$, a trained machine learning model $\mathcal{M}$, and external knowledge of an adversary, denoted by $\mathcal{K}$, a membership inference attack (attack model) can be defined as the following function.

$$
\mathcal{A}: \mathbf{x}_{\text {Target }}, \mathcal{M}, \mathcal{K} \rightarrow\{0,1\}
$$

Here, 0 means $\mathbf{x}_{\text {Target }}$ is not a member of $\mathcal{M}$ 's training dataset $\mathcal{D}_{\text {Train }}$ and 1 otherwise. The machine learning model $\mathcal{M}$ that the adversary targets is also referred to as the target model. As in the work of Shokri et al. [38], we assume the adversary only has black-box access to the target model, such as an MLaaS API, i.e., the adversary can submit a data point to $\mathcal{M}$ and then obtain the probabilistic output, i.e., $\mathcal{M}\left(\mathbf{x}_{\text {Target }}\right)$.

The attack model $\mathcal{A}$ is essentially a binary classifier. Depending on the assumptions, it can be constructed in different ways, which will be presented in later sections.

\section{B. Datasets Description}

We utilize 8 different datasets in this paper to conduct our experiments. Among them, 6 datasets $^{3}$ are the same as the ones used by Shokri et al. [38], i.e., MNIST, ${ }^{4}$ CIFAR-10, CIFAR100, Location [47], Purchase, ${ }^{5}$ and Adult. ${ }^{6}$ We follow the same procedure to preprocess all these datasets.

In particular, the Purchase dataset does not contain any prediction classes. Following Shokri et al. [38], we adopt a clustering algorithm, namely K-means, to manually define classes. The numbers of classes include 2, 10, 20, 50, and 100, therefore, we extend the Purchase dataset into 5 datasets. For instance, Purchase-100 represents the Purchase dataset with 100 different classes.

Moreover, we make use of two other datasets, namely News and Face, in our evaluation. We briefly describe them in the following.

News. The News dataset (20 Newsgroups) is one of the most common datasets used for text classification and clustering. The dataset consists of 20,000 newsgroup documents categorized into 20 classes. The number of data points belonging to each class is very similar, i.e., the dataset has a balanced class distribution. We preprocess the News dataset by first removing headers, footers, and quotes from the documents. Then, we build the TF-IDF matrix out of the raw documents.

Face. The Face dataset (Labeled Faces in the Wild ${ }^{7}$ ) consists of about 13,000 images of human faces crawled from the web. It is collected from 1,680 participants with each participant having at least two distinct images in the dataset. In our evaluation, we only consider people with more than 40 images, which leaves us with 19 people's data, i.e., 19 classes. The Face dataset is challenging for facial recognition, as the images are taken from the web and not under a controlled environment, such as a lab. It is also worth noting that this dataset is unbalanced.

\footnotetext{
${ }^{3}$ We excluded Texas hospital stays dataset [38], as there is not enough information provided for preprocessing it.

${ }^{4}$ http://yann.lecun.com/exdb/mnist/

5 https://www.kaggle.com/c/acquire-valued-shoppers-challenge/data

${ }^{6}$ https://archive.ics.uci.edu/ml/datasets/adult

${ }^{7}$ http://vis-www.cs.umass.edu/lfw/
} 


\section{TOWARDS MOdEL INDEPENDENT MEMBERShIP INFERENCE ATTACKS (ADVERSARY 1)}

In this section, we describe our first adversary considered for membership inference attack. For this adversary, we mainly relax the assumption on her shadow model design. In consequence, membership inference attack can be performed in a much more efficient and less costly way.

We start by defining the threat model. Then, we describe our first simplification, i.e., using one shadow model instead of multiple. In the end, we propose our second simplification which frees the adversary from knowing the target model's structure.

\section{A. Threat Model}

We define our attack model $\mathcal{A}$ as a supervised ML classifier with binary classes (member or non-member). To train $\mathcal{A}$, the adversary needs to derive the labeled training data. i.e., the ground truth membership. As mentioned in Section II, the adversary only has black-box access to the target model, i.e., she is not able to extract the membership status from the target model. Therefore, the adversary trains a shadow model [38] to mimic the behavior of the target model, and relies on the shadow model to obtain the ground truth membership to train $\mathcal{A}$.

To train the shadow model, we assume that the adversary has a dataset, denoted by $\mathcal{D}_{\text {Shadow }}$, that comes from the same underlying distribution as the training data for the target model. Note that most of the experiments by Shokri et al. [38] make the same assumption.

We further assume that the shadow model uses the same ML algorithm and has the same hyperparameters as the target model. To achieve this in practice, the adversary can either rely on the same MLaaS provider which builds the target model or perform model extraction to approximate the target model [43], [30], [45]. Later in this section, we show this assumption can be relaxed as well.

\section{B. One Shadow Model}

Methodology. The adversary's methodology can be organized into three stages, i.e., shadow model training, attack model training, and membership inference.

Shadow Model Training. The adversary first splits her dataset, i.e., $\mathcal{D}_{\text {Shadow }}$, into two disjoint sets, namely $\mathcal{D}_{\text {Shadow }}^{\text {Train }}$ and $\mathcal{D}_{\text {Shadow }}^{\text {Out }}$. Then, she uses $\mathcal{D}_{\text {Shadow }}^{\text {Train }}$ to train her only shadow model, denoted by $\mathcal{S}$.

Attack Model Training. The adversary uses the trained shadow model $\mathcal{S}$ to perform prediction over all data points in $\mathcal{D}_{\text {Shadow }}$ (consisting of $\mathcal{D}_{\text {Shadow }}^{\text {Train }}$ and $\mathcal{D}_{\text {Shadow }}^{\text {Out }}$ ), and obtain the corresponding posterior probabilities. For each data point in $\mathcal{D}_{\text {Shadow }}$, she takes its three largest posteriors (ordered from high to low) or two in the case of binary-class datasets as its feature vector. A feature vector is labeled as 1 (member), if its corresponding data point is in $\mathcal{D}_{\text {Shadow }}^{\text {Train }}$, and as 0 (nonmember) otherwise. All the generated feature vectors and labels are then used to train the attack model $\mathcal{A}$.

Membership Inference. To perform the attack on whether $\mathbf{x}_{\text {Target }}$ is in $\mathcal{D}_{\text {Train }}$, the adversary queries $\mathcal{M}$ with $\mathbf{x}_{\text {Target }}$ to obtain the corresponding posteriors. Then, she picks the 3 maximal posteriors, again ordered from high to low, and feed them into $\mathcal{A}$ to obtain the membership prediction.

It is important to note that our adversary only uses one shadow model and one attack model in her attack, while the approach by Shokri et al. [38] adopts multiple shadow models as well as multiple attack models (one for each class). In particular, as each shadow model is established through MLaaS [38], this strategy will largely reduce the cost of her membership inference attack.

Experimental Setup. We evaluate our attack over all datasets. For each dataset, we first split it by half into $\mathcal{D}_{\text {Shadow }}$ and $\mathcal{D}_{\text {Target }}$. Following the attack strategy, we split $\mathcal{D}_{\text {Shadow }}$ by half into $\mathcal{D}_{\text {Shadow }}^{\text {Train }}$ and $\mathcal{D}_{\text {Shadow }}^{\text {Out }} . \mathcal{D}_{\text {Target }}$, on the other hand, is used for attack evaluation, it is also split by half: One is used to train the target model, i.e., $\mathcal{D}_{\text {Train }}$, and serves as the members of the target model's training data, while the other serves as the non-member data points.

For image datasets, i.e., MNIST, CIFAR-10, CIFAR-100, and Face, we use convolutional neural network (CNN) to build the target model. Our CNN is assembled with two convolutional layers and two pooling layers with one hidden layer containing 128 units in the end. For the other datasets, we use multilayer perceptron (neural network) with one hidden layer (128 units) as the target model. Each shadow model's structure is the same as its corresponding target model, following the assumption that the adversary knows the target model's structure. The attack model is established with another multilayer perceptron (a 64-unit hidden layer and a softmax output layer). All our experiments are implemented in Python with Lasagne. ${ }^{8}$ For reproducibility purposes, our code is available at https://github.com/AhmedSalem2/ML-Leaks.

We compare our attack against the attack by Shokri et al. [38]. Following the original configuration of the authors' code, ${ }^{9}$ we train 10 shadow models and multiple attack models (one for each class).

As membership inference is a binary classification, we adopt precision and recall as our evaluation metrics. Moreover, we use accuracy to measure the target model's prediction performance.

Results. Figure 1 depicts the first adversary's performance. In general, we observe that our attack has a very similar membership inference as the previous work [38]. For instance, our attack on the CIFAR-100 dataset achieves 0.95 for both precision and recall, while the attack by Shokri et al. has a 0.95 precision and 0.94 recall. It is also interesting to see that our attack works for both balanced datasets, such as CIFAR-10, and unbalanced datasets, such as Face.

We also observe variations of the attack performance on different datasets. We relate this to the overfitting level of ML models on different datasets, similar to previous works [38], [48]. We quantify the overfitting level of a target model as the difference between its prediction accuracy on the training set and testing set. Through investigation, we discover that if an ML model is more overfitted, then it is more vulnerable

\footnotetext{
${ }^{8}$ https://github.com/Lasagne/Lasagne

${ }^{9}$ https://github.com/csong27/membership-inference
} 


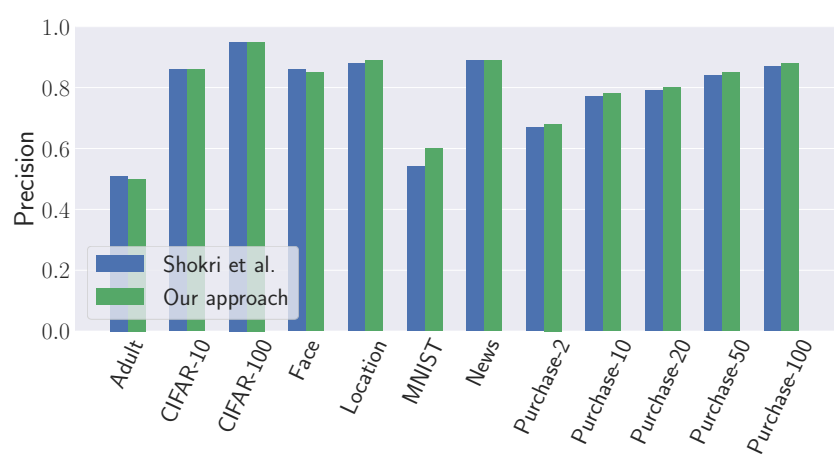

(a) Precision.

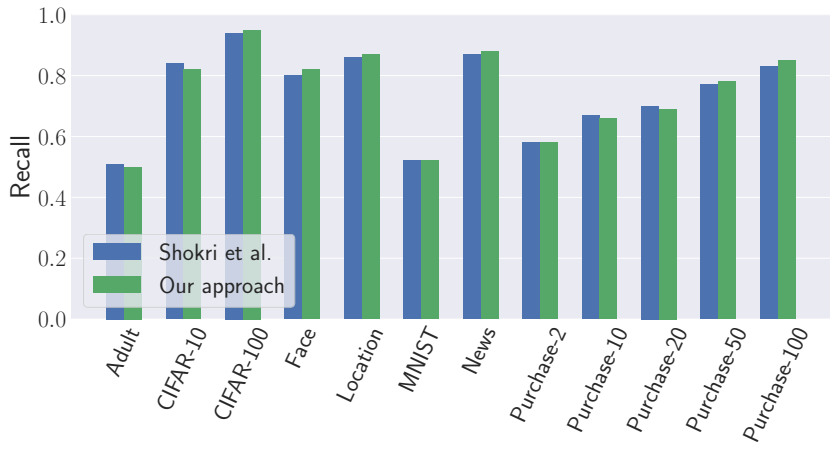

(b) Recall.

Fig. 1: Comparison of the first adversary's performance with Shokri et al.'s using all datasets. (a) precision, (b) recall.

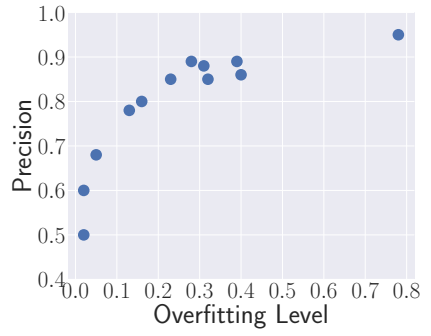

(a)

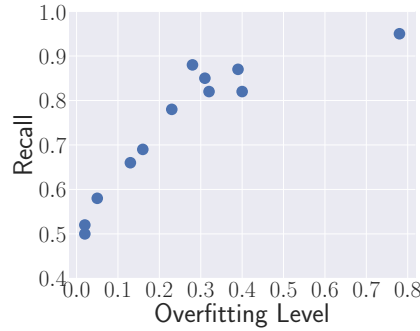

(b)
Fig. 2: The relation between the overfitting level of the target model measured by the difference between prediction accuracy on training set and testing set (x-axis) and membership inference attack performance (y-axis). (a) precision, (b) recall.

to membership inference attack (see Figure 2). For instance, our attack on the Adult dataset achieves a relatively weak performance (around 0.5 precision and recall), and there is only a $2 \%$ difference between the target model's training and testing accuracy. On the other hand, the membership inference attack achieves a 0.95 precision and recall on the CIFAR-100 dataset. Meanwhile, the corresponding target model provides a much better prediction performance on the training set than on the testing set, i.e., $78 \%$ difference.

To further demonstrate the relationship between overfitting and membership inference, we perform another - more controlled - experiment on the Location and Purchase-100 datasets. Concretely, we focus on the number of epochs used in training, larger number leads to higher overfitting. We vary the number of epochs used from 10 to 100 and report the result in Figure 3. As we can see, the attack performance indeed increases with the increase of number of epochs.

Another factor which also affects our attack's performance is the number of classes in the dataset. Both CIFAR-10 and CIFAR-100 are image datasets with different number of classes (10 vs 100), it turns out that our membership inference attack on the latter dataset achieves a $10 \%$ better performance than on the former dataset. Similar results can be observed from all the Purchase datasets.

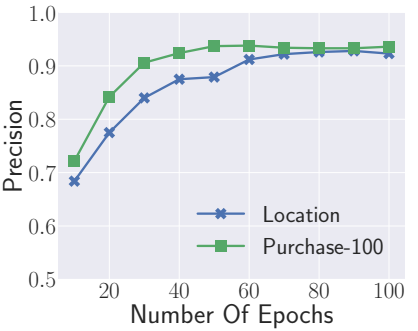

(a)

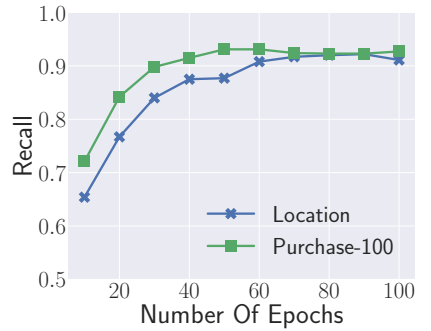

(b)
Fig. 3: The relation between the number of epochs used during the training of the target model (x-axis) and membership inference attack performance (y-axis). (a) precision, (b) recall.

For our attacks, we use only the three highest posterior probabilities (in descending order) as the features for our attack. We test the effect of using more posteriors on the CIFAR-100, Location, MNIST, and News datasets. The result in Figure 4 shows that this factor does not have a significant effect on the attack's performance for most of the datasets. Generally, three posteriors achieves the best performance, especially on the MNIST dataset.

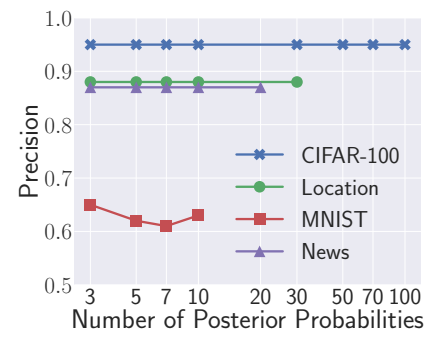

(a)

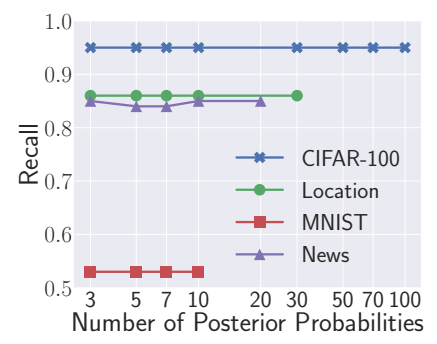

(b)
Fig. 4: The effect of the number of posterior probabilities (used as features) on the first adversary's performance. (a) precision, (b) recall.

A major difference between our attack and the previous 


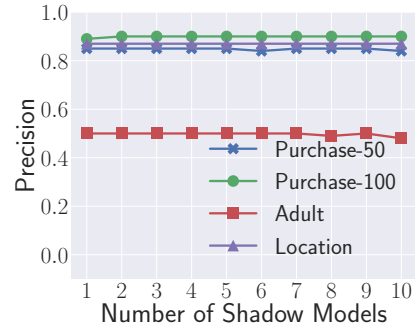

(a)

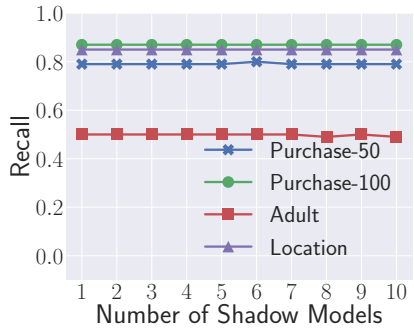

(b)
Fig. 5: The effect of the number of shadow models on the first adversary's performance. (a) precision, (b) recall.

one [38] is the number of shadow models used. We further study this factor's influence on our own attack's performance. Figure 5 shows the corresponding results on the Purchase100, Purchase-50, Adult, and Location datasets. By varying the number of shadow models from 1 to 10 , we do not observe a significant performance difference for both precision and recall. This means increasing the number of shadow models does not improve our attack's performance.

Evaluation on MLaaS. All the above experiments are conducted in a local setting. We further evaluate our attack with a real-world MLaaS. In particular, we use Google's MLaaS, namely Google Cloud Prediction API. ${ }^{10}$ Under this service, a user can upload her own data and get the black-box ML API trained by Google. The user can neither choose which classifier to use, nor the corresponding model structure and parameters. We perform our attack following the same methodology as in Section III-B. We construct both target model and shadow model with Google's MLaaS and build our attack model locally.

We use the Purchase-100 and Location datasets for evaluation and observe that the attack's performance is even stronger than our previous local evaluation. For the Purchase-100 dataset, our attack on Google's MLaaS has a 0.90 precision and a 0.89 recall, while our local evaluation has a 0.89 precision and a 0.86 recall. For the Location dataset, the precision is 0.89 and the recall is 0.86 , which is almost similar to our local evaluation ( 0.88 precision and 0.86 recall).

\section{Target Model Structure}

One of the above attack's assumptions is that the adversary knows the target model's algorithm and hyperparameters and implements her shadow model in the same way. Next, we show how to relax this assumption. We first concentrate on target model's hyperparameters, then, the type of classifiers it uses.

Hyperparameters. We assume that the adversary knows the target model is a neural network, but does not know the details of the model. We first train the shadow model with half of the training parameters of the target model. More precisely, we reduce the batch size, hidden units, and regularization parameters to half. On the Purchase-100 dataset, our attack

\footnotetext{
${ }^{10}$ Google's Cloud prediction API is deprecated on April 20th, 2018 (https: //cloud.google.com/prediction/), the new MLaaS provided by Google is called Cloud Machine Learning Engine.
}

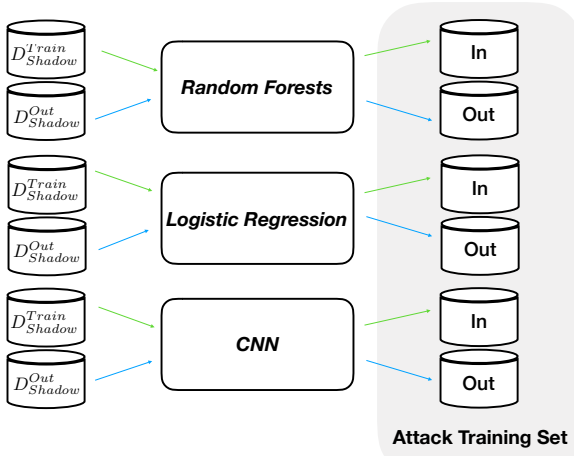

Fig. 6: The architecture of the combining attack on generating data for training the attack model.

achieves a 0.86 precision and 0.83 recall, which is almost the same as the one reported in Figure 1. We also revert the settings to test the case when the shadow model has double number of parameters than the target model. The performance drops a bit to 0.82 precision and 0.80 recall, but it is still quite close to our original attack. We also perform evaluation over other datasets and observe similar results. This evaluation shows the flexibility of the membership inference attack: An adversary with no knowledge about the model's hyperparameters can still get good performance.

Target Model's Algorithm. We further assume that the adversary has no knowledge on what classification algorithm is adopted by the target model. In this setting, our first attempt is to use any classifier, such as random forests, as the shadow model and attack the target model that is (very likely to be) different from the shadow model, such as CNN. However, the experimental results are not very promising.

To improve the attack with no knowledge of the target model, we construct a set of ML models, each with a different classification algorithm, and combine them together as one shadow model. Each single ML model is referred to as a subshadow model. This is achievable as the types of classifiers are limited. This attack, also referred to as the combining attack, can learn the behavior of the different classifiers and therefore can attack an unknown target model based on the assumption that there is a sub-shadow model which is trained with the same classifier as the target model.

Concretely, we use the same methodology as in Section III-B to train multiple sub-shadow models as illustrated in Figure 6, with each sub-shadow model being a different classifier. The data each sub-shadow model is trained on is the same. All the generated features by all sub-shadow models are stacked together, i.e., the attack model $\mathcal{A}$ is trained with a larger dataset. In this new dataset, each data point in $\mathcal{D}_{\text {Shadow }}$ is represented multiple times with respect to different subshadow models' outputs.

We run a local experiment on the Purchase-100 dataset to evaluate this attack. Three popular ML classifiers, i.e., multilayer perceptron, random forests (with 1,000 tree), and logistic regression are adopted as sub-shadow models. The target model for the Purchase-100 dataset is a multilayer perceptron. For a more complete comparison, we further build 


\begin{tabular}{lcccccc}
\hline \multirow{2}{*}{ Classifier } & \multicolumn{2}{c}{ With target model structure } & & \multicolumn{2}{c}{ Combining attack } \\
\cline { 2 - 3 } \cline { 6 - 7 } & Precision & Recall & & Precision & Recall \\
\hline Multilayer perceptron & 0.86 & 0.86 & & 0.88 & 0.85 \\
Logistic regression & 0.90 & 0.88 & & 0.90 & 0.88 \\
Random forests & 1.0 & 1.0 & & 0.94 & 0.93 \\
\hline
\end{tabular}

TABLE II: Comparison of the combining attack and the original attack by the first adversary proposed in Section III-B.

another two target models that are based on random forests and logistic regression, respectively, and use the same algorithm to build a single shadow model as in Section III-B. Table II depicts the result. As we can see, our combining attack has a similar performance when target model is multilayer perceptron and logistic regression. Meanwhile, the attack's performance is relatively worse is when the target model is random forests.

In conclusion, we show that our combining attack can free the attacker from knowing the target model, which further enlarges the scope of membership inference attack.

\section{TOWARDS DATA INDEPENDENT MEMBERSHIP INFERENCE ATTACKS (ADVERSARY 2)}

In this section, we relax the assumption on the adversary having a dataset that comes from the same distribution as the target model's dataset.

We start by explaining the threat model, then describe the adversary's attack methodology. In the end, we present a comprehensive experimental evaluation.

\section{A. Threat Model}

Different from the threat model in Section III, we remove the assumption that the adversary has a dataset $\mathcal{D}_{\text {Shadow }}$ coming from the same distribution as the training data for the target model. This largely reduces the attack capabilities of the adversary. For this scenario, Shokri et al. [38] propose to query the target model multiple times to generate synthetic data to train the shadow model. However, this approach can only be applied when the dataset is assembled with binary features. ${ }^{11}$ In contrast, our approach can be applied to attack ML models trained on any kind of data.

\section{B. Methodology}

The strategy of the second adversary is very similar to the one of the first adversary. The only difference is that the second adversary utilizes an existing dataset that comes from a different distribution than the target model's training data to train her shadow model. We refer to this attack as the data transferring attack.

The shadow model here is not to mimic the target model's behavior, but only to summarize the membership status of a data point in the training set of a machine learning model. As only the three - or two in case of binary datasets - largest posteriors are used for the attack model, we can also neglect the effect brought by datasets with different number of classes.

\footnotetext{
${ }^{11}$ We confirm this with the authors.
}

\section{Evaluation}

Experimental Setup. We use the same attack model and shadow model setup as presented in Section III, such as data splitting strategy and the types of ML models used. We perform the data transferring attack over all datasets. For evaluation metric, we again use precision and recall.

Results. Figure 7 depicts the data transferring attack's performance. The $\mathrm{x}$-axis represents the dataset being attacked, i.e., the dataset the target model is trained on, and the y-axis represents the dataset used for training the shadow model. Compared to the first adversary the attack results of which are listed at the diagonal of Figure 7, the second adversary in multiple cases obtains similar performances. For instance, using the Face dataset to attack the CIFAR-100 dataset results in 0.95 for both precision and recall, while the corresponding results for the first adversary are also 0.95 for both metrics. In several cases, we even observe a performance improvement over the first adversary. For instance, using the Purchase-10 dataset to attack the News dataset achieves a 0.93 precision and 0.92 recall, while the first adversary has a 0.88 precision and 0.86 recall. More interestingly, in many cases, datasets from different domains can effectively attack each other, e.g., the News dataset and the CIFAR-100 dataset.

For the first adversary, we relax the assumption on shadow model design. This relaxation also applies for the second adversary, as the shadow model and target model are trained with different datasets. For instance, the Purchase-20 dataset is trained with a multilayer perceptron while the CIFAR-100 dataset is trained with a CNN.

One of the major advantages of our data transferring attack lies in its applicability. The synthetic data generation strategy by Shokri et al. [38] cannot be applied to dataset of any kind, but those with binary features. Even for dataset of binary features, a single synthetic data point requires 156 queries [38] to the target model. Given the large dataset quantity needed for ML models and MLaaS's pay-per-query business model, this is very costly. Moreover, sending a large amount of queries to an MLaaS API would alert the server, which may not even allow the adversary to finish her synthetic data generation process. Meanwhile, our data transferring attack does not have any of the above constraints.

Reasoning. After demonstrating the strong performance of our data transferring attack, we now seek to understand the reason behind. To this end, we pick the highest three posteriors (similar to our attack) of member and non-member data points with respect to their target ML models of all datasets, and embed these posteriors into a 2D space using t-Distributed Stochastic Neighbor Embedding (t-SNE). We show in Figure 8a the result for two datasets (of different types) between which our transferring attack is effective. As we can see, the member and non-member points of these datasets are tightly clustered together and follow a common decision boundary, thus, the attack model trained on one dataset can effectively infer the membership status of points in the other dataset. Meanwhile, Figure $8 \mathrm{~b}$ shows the results for two datasets between which our transferring attack is not effective. As depicted, there are no clear clusters for members and non-member data points. 


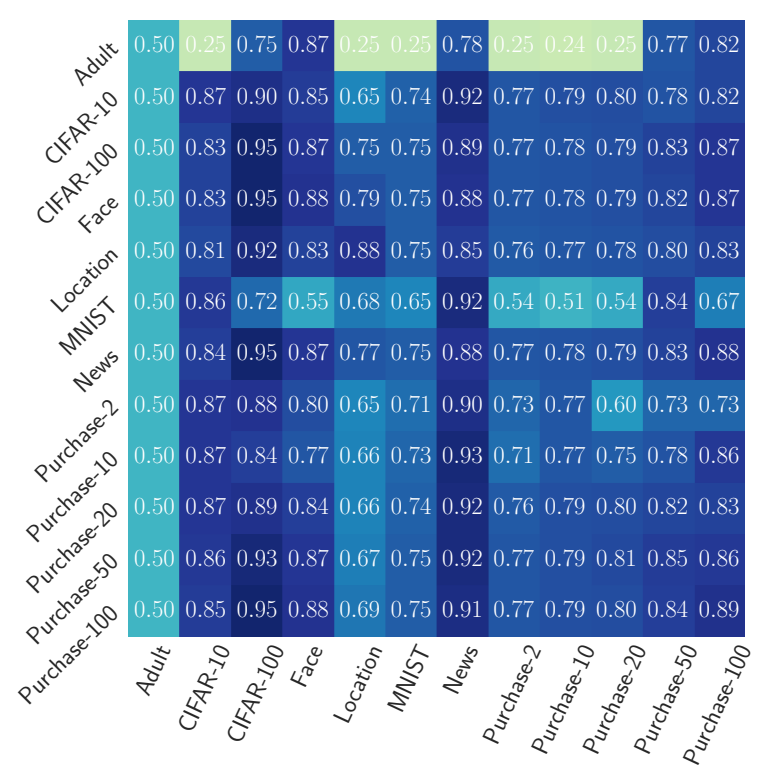

(a) Precision

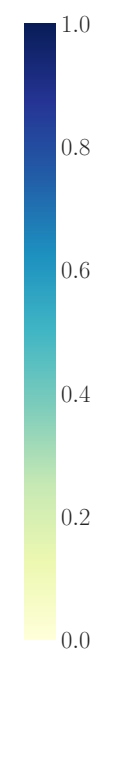

Fig. 7: The performance of our data transferring attack. The x-axis represents the dataset being attacked, i.e., the dataset the target model is trained on. The $y$-axis represents the dataset used for training the shadow model.

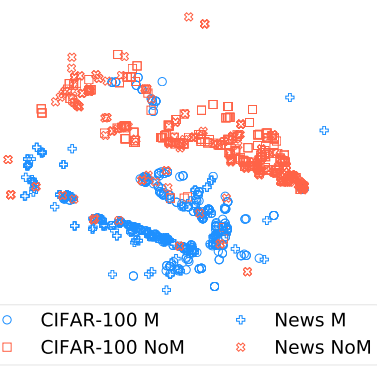

(a)

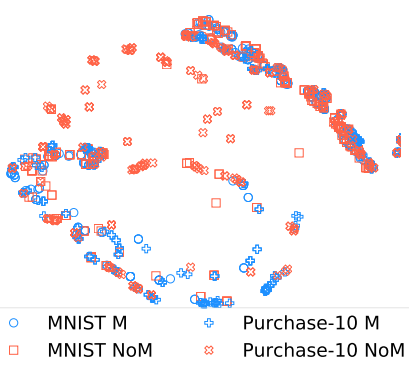

(b)
Fig. 8: The top three posteriors of member and non-member data points (a random sample) projected into a 2D space using t-Distributed Stochastic Neighbor Embedding (t-SNE). (a) CIFAR-100 and News, (b) MNIST and Purchase-10. M means member and NoM means non-member.

\section{Evaluation On MLaaS}

We also evaluate our data transferring attack on Google's MLaaS. Concretely, we use a shadow model trained on the Location dataset to attack a target model trained on the Purchase-100 dataset. Both models are trained with Google's MLaaS. Experimental results show that we achieve a 0.8 precision and 0.78 recall. By further flipping the shadow and target model, i.e., Purchase-100 dataset attacking Location dataset, the membership inference result is still very strong with a 0.87 precision and a 0.82 recall. This shows that our data transferring attack is not only effective in the local setting but also in the real-world MLaaS setting.

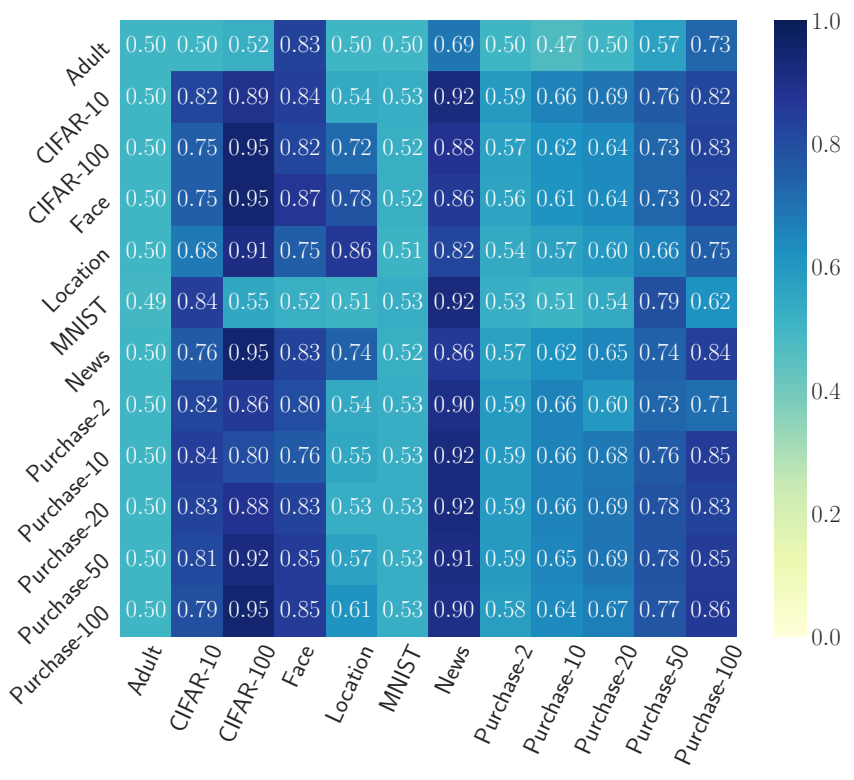

(b) Recall

\section{MOdel AND DATA IndePENDENT MEMBERShiP INFERENCE ATTACK WITHOUT TRAINING (ADVERSARY 3)}

In this section, we present our third adversary, who does not need to train any shadow model and does not assume knowledge of model or data distribution. We start with the threat model description. Then, we list the attack methodology. In the end, we present the evaluation results.

\section{A. Threat Model}

We relax the assumption that the adversary needs to train any shadow model to perform her attack. All she could rely on is the target model's output posteriors $\mathcal{M}\left(\mathbf{x}_{\text {Target }}\right)$ after querying her target data point $\mathbf{x}_{\text {Target }}$. Note that Yeom et al. [48] propose a similar attack, however, their membership inference attack requires the adversary to know the target data point's class label which is hard to obtain in some cases, such as in biomedical settings [4]. Therefore, our threat model covers a broader range of scenarios.

\section{B. Methodology}

The attack model for the third adversary is implemented as an unsupervised binary classification. Concretely, the adversary first obtains $\mathcal{M}\left(\mathbf{x}_{\text {Target }}\right)$. Then, she extracts the highest posterior and compares whether this maximum is above a certain threshold. If the answer is yes, then she predicts the data point is in the training set of the target model and vice versa. The reason we pick maximum as the feature follows the reasoning that an ML model is more confident, i.e., one posterior is much higher than others, when facing a data point that it was trained on. In another words, the maximal posterior of a member data point is much higher than the one of a non-member data point. 


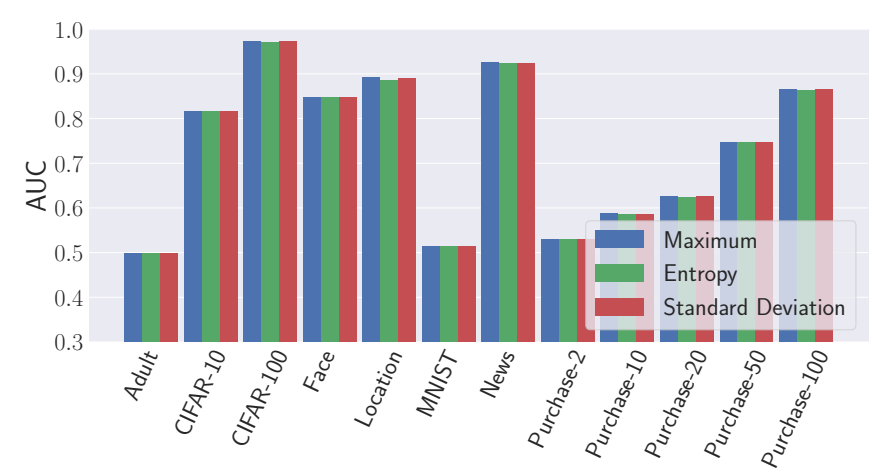

Fig. 9: The AUC values for three different statistical measures over all datasets. We include the results for the Adult and News datasets as AUC is independent of a concrete detection threshold.

Threshold Choosing. The attacker can pick the threshold for membership inference depending on her requirements, as in many machine learning applications [50], [3]. For instance, if she concentrates more on inference precision (recall), then she can pick a relatively high (low) threshold.

Nevertheless, we provide a general method for choosing a threshold. Concretely, we generate a sample of random points in the feature space of the target data point. For image datasets including CIFAR-10, CIFAR-100, MNIST, and Face, we generate random images, where the value of each pixel is drawn from a uniform distribution. For datasets with binary features including Location and Purchase datasets, we generate 0 and 1 for each feature according to an unbiased coin flip. For Adult and News, as the bounds for features are not clear, our method cannot apply. One way to tackle this is to collect News articles or people's records (with the same features as in the Adult dataset) from the Internet as the "random" points. We leave this for future work. Next, we query these random points to the target model to get the corresponding maximal posteriors. We hypothesize that these points act as the nonmember points. Thus, top $t$ percentile of these random points' maximal posteriors can serve as a good threshold. Below, we show empirically that there exists a choice of $t$ percentile that works well and generalizes across all the dataset and therefore can be used to automatically determine the detection threshold.

\section{Evaluation}

Experimental Setup. We evaluate the third adversary over all datasets except News and Adult. Note that we do not need to split the dataset as this adversary does not train any shadow model. Instead, we split each dataset by half, and use one part to train the target model and the other part is left out as nonmembers.

Results. We first evaluate the effectiveness of maximal posterior on differentiating member and non-member points without setting a threshold. To this end, we adopt the AUC (area under the ROC curve) value, which reports the relation between true positive rate and false negative rate over multiple thresholds, as the evaluation metric [13], [3], [35], [32], [49]. Besides maximal posterior probability, we further test the effect of using other statistical metrics, including standard deviation and

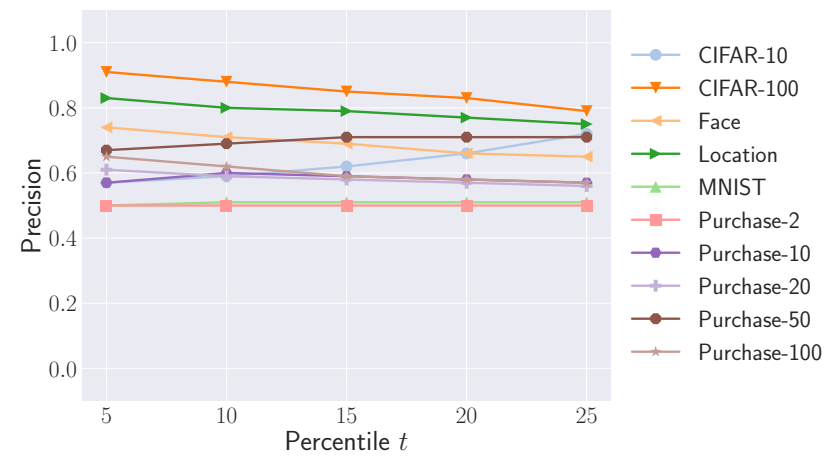

(a)

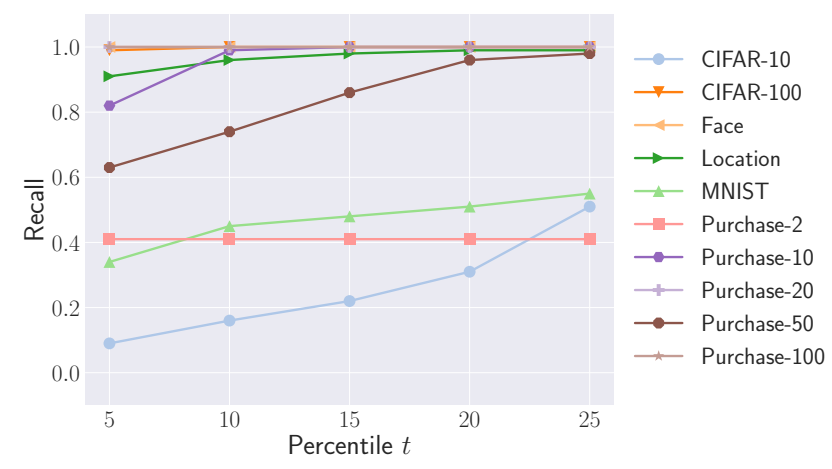

(b)

Fig. 10: The relation between the percentile of the maximal posterior, i.e., threshold, (x-axis) and the third adversary's performance (y-axis). (a) precision, (b) recall.

entropy. In particular, the entropy of posteriors is defined as $-\sum_{p_{i} \in \mathcal{Y}} p_{i} \log p_{i}$, where $p_{i}$ denotes the posterior for the $i$ th class. Figure 9 shows that maximal posterior achieves very high performance: In multiple datasets, we obtain AUC values above 0.8. Meanwhile, the AUC score is almost the same for all the three measures. This indicates that standard deviation and entropy can also be used as features for the attack.

Next, we evaluate our concrete prediction following our threshold-choosing method. We generate 1,000 random data points for each dataset and experiment multiple thresholds with respect to the top $t$ percentile. Figure 10 shows the results. As we can see, setting $t$ to 10 achieves a good performance (both precision and recall) for most of the datasets, such as CIFAR-100. Figure 11a further shows the maximal posterior distribution of member, non-member, and random points for CIFAR-100. As the figure shows, our random points' maximal posteriors behave similarly to the distribution of the non-member points' which leads to the strong membership inference. On the other hand, our attack does not perform well on some datasets, such as Purchase-10, the corresponding maximal posteriors of which are shown in Figure 11b.

We also experiment with picking a fixed threshold for membership inference, e.g., maximal posterior above $50 \%$. However, the evaluation shows that there is no single number that can achieve good performance for all the datasets. Thus, we conclude that our threshold-choosing method is suitable. 


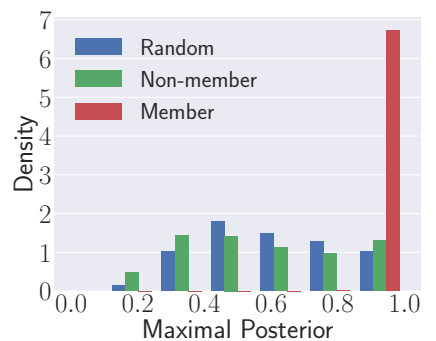

(a) CIFAR-100

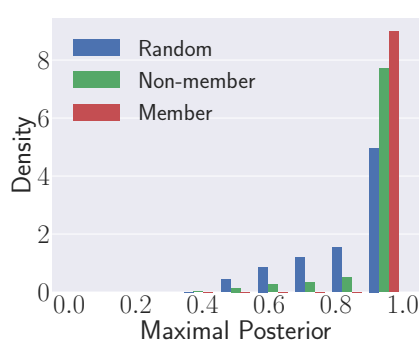

(b) Purchase-10
Fig. 11: The distribution of the maximal posterior of the ML model for the member, non-member, and random points for two datasets: (a) CIFAR-100, (b) News. The y-axis shows the density.

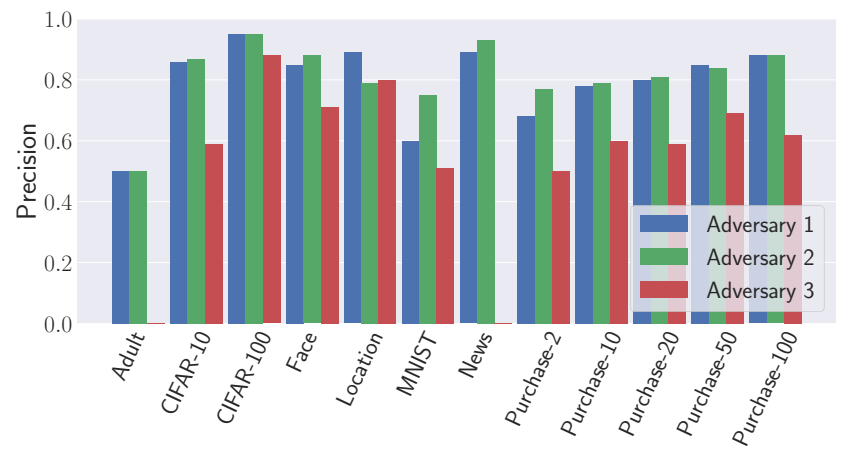

(a)

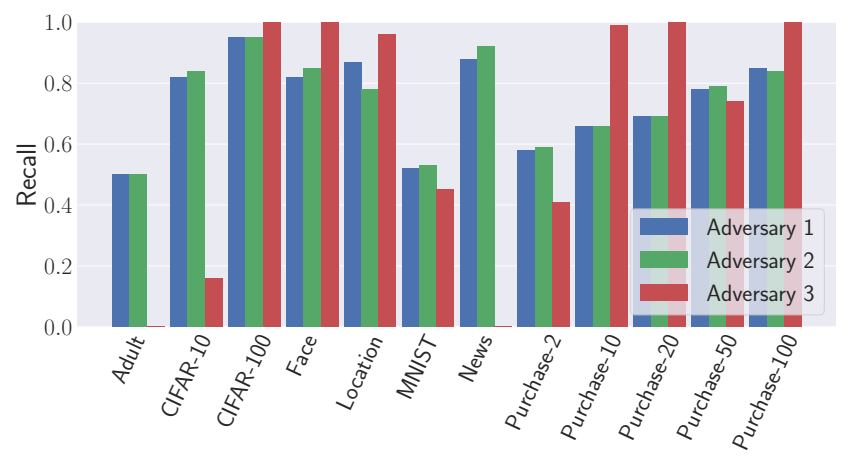

(b)

Fig. 12: Comparison of the three different adversaries' performance. (a) precision, (b) recall.

\section{Comparison of the Three Attacks}

Figure 12 compares the performance, i.e., precision and recall, of the three attacks. ${ }^{12}$ In particular, we show the best performance for our data transferring attack (adversary 2). As we can see, our first two adversaries achieve very similar performance for most of the datasets. On the other hand, the performance of our third adversary with the minimal assumptions is only slightly worse (especially for precision). These results clearly demonstrate membership inference attacks are

\footnotetext{
${ }^{12}$ All the comparisons are done on the same dataset setting.
}

very broadly applicable, thereby the corresponding risks are much more severe than previously shown.

\section{DEFENSE}

In this section, we propose two defense techniques aiming at mitigating membership privacy risks. The effectiveness of our membership inference attacks is mainly due to the overfitting nature of ML models. Therefore, our defense techniques are designed to increase ML models' generalizability, i.e., prevent them from being overfitted.

Our first technique is dropout which is designed for neural network-based classifiers. ${ }^{13}$ Our second technique is model stacking. This mechanism is suitable for all ML models, independent of the classifier used to build them.

As the first and second adversaries follow the same methodology of building a shadow model (with different assumptions on the dataset), we only show the effectiveness of our defense on the first adversary as well as on the third adversary to conserve space. For the first adversary, to fully assess the attack's performance under our defense, we further assume the attacker knows the defense technique being implemented and builds her shadow model following the same defense technique.

\section{A. Dropout}

Methodology. A fully connected neural network contains a large number of parameters which is prone to overfitting. Dropout is a very effective method to reduce overfitting based on empirical evidences. It is executed by randomly deleting in each training iteration a fixed proportion (dropout ratio) of edges in a fully connected neural network model. We apply dropout for both the input layer and the hidden layer (see Section III) of the target model. We set our default dropout ratio to be 0.5 .

Evaluation. We test dropout on all datasets against the first adversary and third adversary (except for the News and Adult datasets). Figure 13a and Figure 13b compare the performance of the first adversary's performance before and after the dropout defense. As we can see, the attack performance is reduced in almost all cases. For instance, the precision of the attack on the Purchase-100 dataset drops from 0.89 to 0.64 , while the recall drops from 0.86 to 0.63 . In another example, the precision and recall on the CIFAR-100 dataset drop by more than $30 \%$. There is only one case where dropout does not help much, i.e., the target model trained on the News dataset.

Similarly, the performance of our third adversary is reduced due to dropout (see Figure 14). For example, the precision and recall of the attack on the CIFAR-100 dataset drop by more than $25 \%$ and $40 \%$, respectively. However, on some datasets, such as MNIST, the recall of the attack even improves. This indicates that our third adversary is more resistant to dropout than our first adversary.

Figure 15 further shows the original target model's performance (prediction accuracy) after dropout has been applied.

\footnotetext{
${ }^{13}$ Note that for different kinds of classifiers, dropout can be replaced by other regulation techniques, such as the $L_{2}$-norm, which is also shown to be effective against the membership inference attack [38].
} 


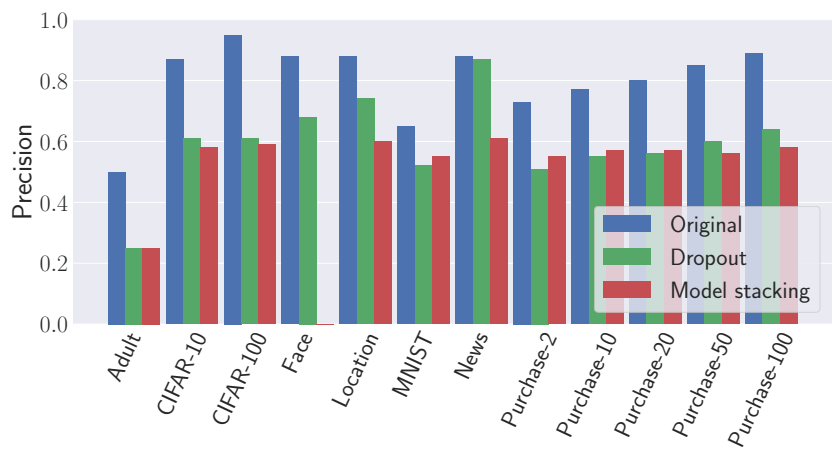

(a)

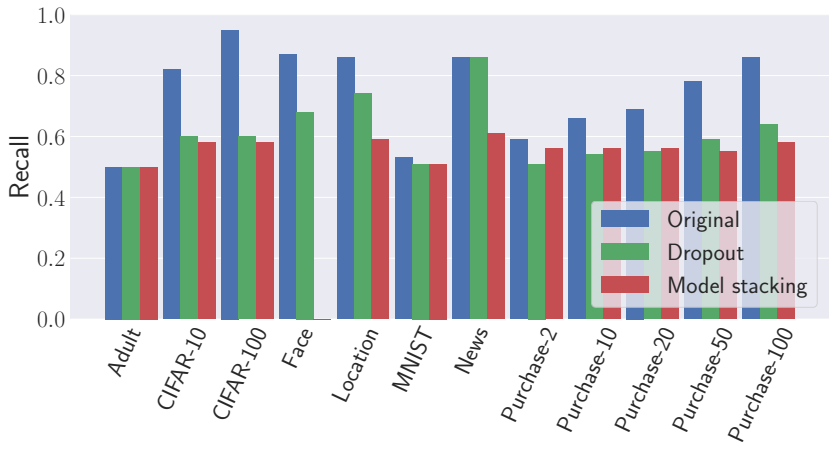

(b)

Fig. 13: Comparison of the first adversary's performance under both of the defense mechanisms. (a) precision, (b) recall.

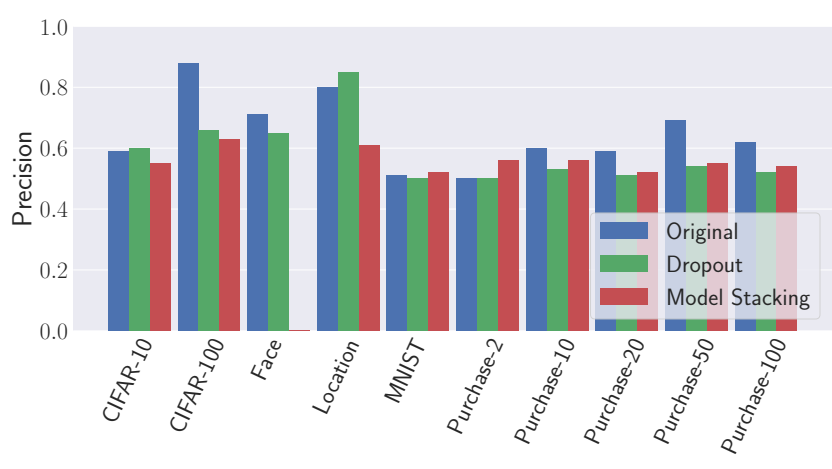

(a)

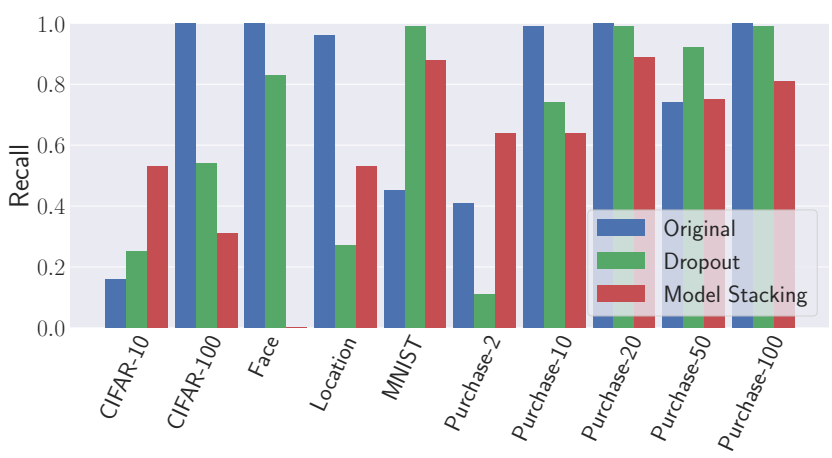

(b)

Fig. 14: Comparison of the third adversary's performance under both of the defense mechanisms. (a) precision, (b) recall.

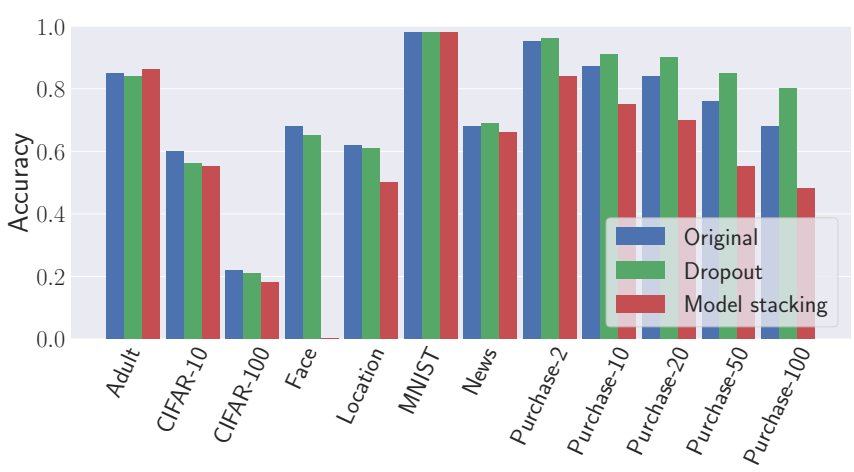

Fig. 15: Comparison of the target model's accuracy under both of the defense mechanisms.

We observe that, on more than half of the datasets, the dropout mechanism even increases the target model's prediction performance. For instance, on the Purchase-50 dataset, the target model's accuracy increases from 0.72 to 0.83 .

Figure 16 plots the relation between the overfitting level (see Section III) reduction and the first adversary's performance reduction after dropout has been applied. The overfitting level reduction is calculated as the original target model's over-

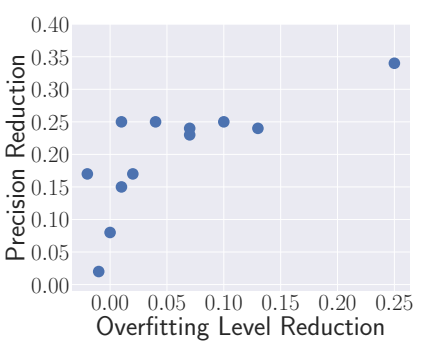

(a)

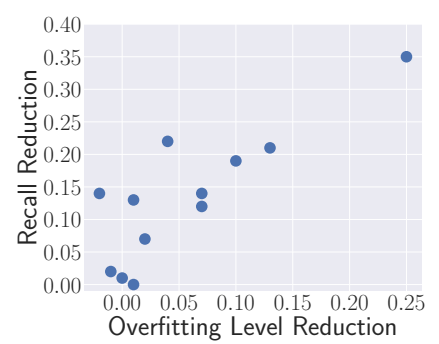

(b)
Fig. 16: The relation between the overfitting level reduction (x-axis) and the first adversary's performance reduction ( $y$ axis) when applying dropout as the defense mechanism. (a) precision reduction, (b) recall reduction.

fitting level subtracting the dropout-defended target model's overfitting level. As we can see, more effective dropout which results in larger reduction on overfitting level leads to better defense against membership inference attacks. These results support the argument by Shokri et al. [38] that overfitting is a common enemy for the membership privacy risks and the target model's performance. 


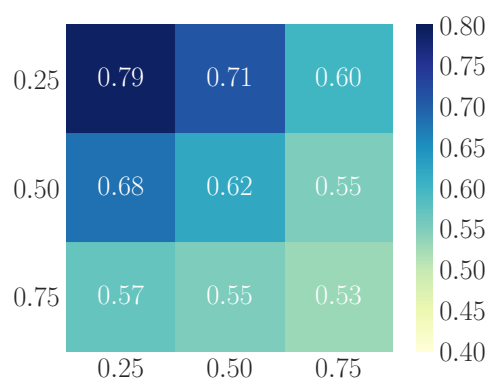

(a) Precision

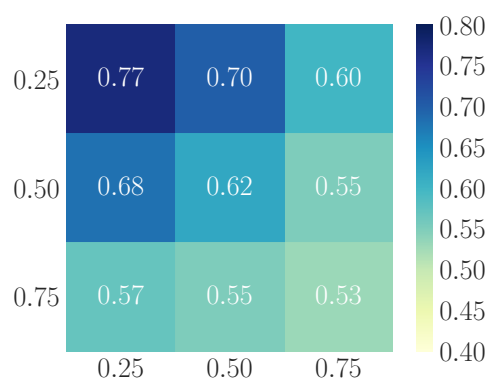

(b) Recall

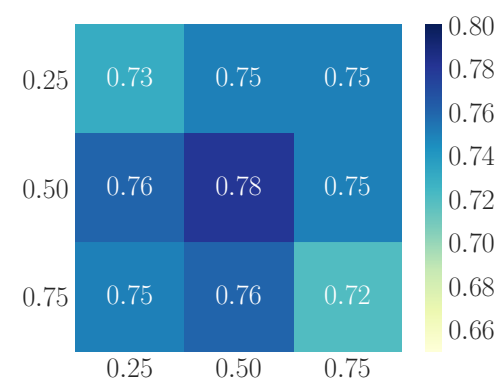

(c) Accuracy

Fig. 17: The effect of the dropout defense on the first adversary's performance, i.e., (a) precision and (b) recall, and on (c) the target model's accuracy under different dropout ratios in different layers of the neural network. The x-axis represents the input layer, and the y-axis represents the hidden layer.

So far, we have used 0.5 as the dropout ratio. We further test the effect of varying the dropout ratio of our defense. We try different dropout ratios on both input and fully connected layers while monitoring the results on the first adversary's performance and the target model's accuracy. Figure 17 shows the result on the Purchase-100 dataset. We first observe that higher dropout ratio leads to lower attack performance. For instance, dropout ratio 0.75 on both layers reduces the attack's performance to 0.53 precision and recall. On the other hand, both large and small dropout ratio result in the low performance of the target model. This means the accuracy of the target model is the strongest when dropout ratio is mediate. In conclusion, 0.5 dropout ratio is a suitable choice for this defense technique.

\section{B. Model Stacking}

Methodology. The dropout technique is effective, however, it can only be applied when the target model is a neural network. To bypass this limitation, we present our second defense technique, namely model stacking, which works independently of the used ML classifier.

The intuition behind this defense is that if different parts of the target model are trained with different subsets of data, then the complete model should be less prone to overfitting. This can be achieved by using ensemble learning.

Ensemble learning is an ML paradigm, where instead of using a single ML model, multiple ML models are combined to construct the final model. There are different approaches to combine these ML models, such as bagging or boosting. For our defense, we focus on stacking the models in a hierarchical way. Figure 18 shows a sample architecture for model stacking.

Concretely, we organize the target model in two layers over three ML models. The first layer consists of two ML models (the first and second model). The second layer consists of a single ML model (the third model). As shown in Figure 18, to get the model's output on some data point $\mathbf{x}$, we first apply $\mathbf{x}$ on each of the first two models to have their posteriors $\mathcal{Y}^{1}$ and $\mathcal{Y}^{2}$. We then concatenate both outputs, i.e., $\mathcal{Y}^{1} \| \mathcal{Y}^{2}$, and apply the result to the third model which predicts the final output $\mathcal{Y}$.
To maximize the prevention of overfitting, we train the three different models on disjoint sets of data. The intuition behind is that there is no data point seen by more than one model during training.

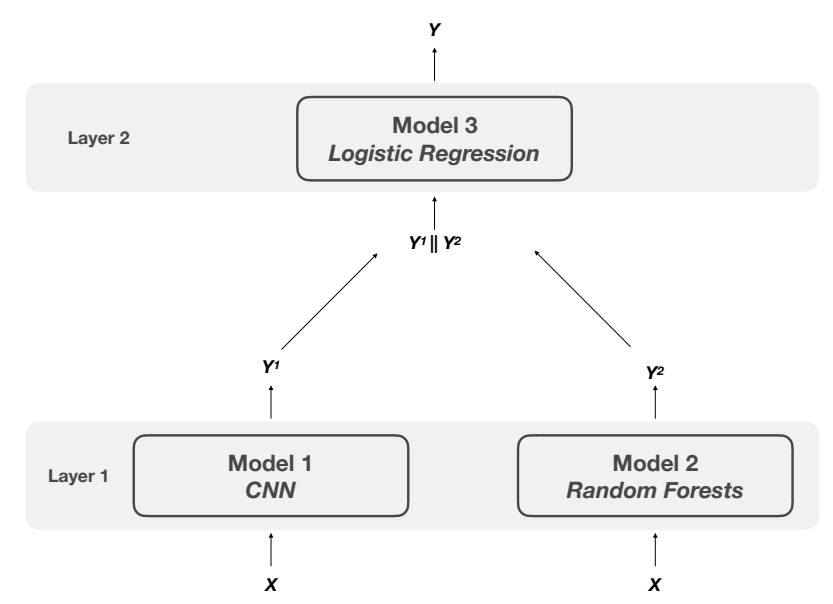

Fig. 18: The architecture of model stacking.

Evaluation. For our evaluation, we use multilayer perceptron or $\mathrm{CNN}$ as the first model, random forests as the second model, and logistic regression as the third model. We pick this architecture to test the effect of using different machine learning models in the different layers. However, a different selection of models also suffices.

We build both target and shadow models for the first adversary as described, i.e., each model consists of 3 different ML models. To train our target and shadow models, we split the data into 12 disjoint sets. We use the first 6 sets to train and test our target model, and the remaining 6 to train and test the shadow model.

We evaluate this technique on all datasets but the Face dataset as it does not have enough data to provide meaningful results in this setting. Figure 13 shows the result for the first adversary. As we can see, model stacking reduces the attack's performance significantly in all cases. For instance, 


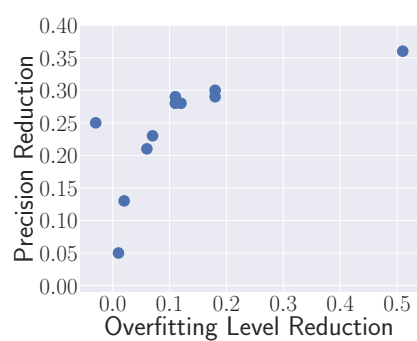

(a)

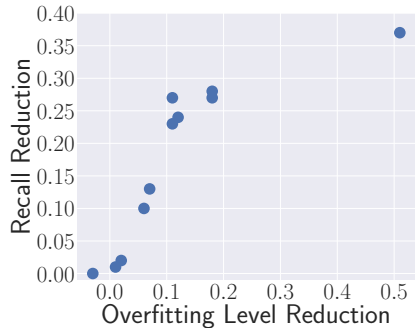

(b)
Fig. 19: The relation between the overfitting level reduction (xaxis) and the first adversary's performance reduction (y-axis) when applying model stacking as the defense mechanism. (a) precision reduction, (b) recall reduction.

on the CIFAR-10 dataset, model stacking reduces the attack's precision and recall by more than $30 \%$. Moreover, compared to the dropout defense, model stacking is more effective in some cases. Dropout does not change the attack's performance on the News dataset while model stacking reduces the corresponding precision and recall by $28 \%$. The same result can be observed on the Location dataset. However, model stacking affects target model's accuracy more than dropout in multiple cases, e.g., the Purchase datasets. The relation between overfitting level reduction and attack performance reduction for the model stacking technique is very similar to the one for the dropout technique, the results are depicted in Figure 19.

Similarly, the performance of our third adversary drops after model stacking has been applied (see Figure 14). For instance, model stacking reduces the attack's performance on the Location dataset by more than $20 \%$ for the precision and $30 \%$ for the recall. But similar to the dropout defense, exceptions like MNIST also exist.

In conclusion, if the target model is not based on neural networks, model stacking is an effective defense technique. Otherwise, dropout is sufficient to mitigate the membership privacy risks due to its high utility maintenance.

\section{RELATED WORK}

Membership Inference. Membership inference attack has been successfully performed in many different data domains, ranging form biomedical data [18], [2] to mobility traces [35].

Homer et al. [18] propose the first membership inference attack on genomic data. This attack relies on the $L_{1}$ distance between the allele frequencies and the victim's genomic data. Backes et al. [2] generalize this attack to other types of biomedical data. More recently, Pyrgelis et al. [35] have shown that people's aggregate mobility traces are also prone to membership inference attack. They first formalize membership inference as a distinguishability game. Then, they implement the attack with machine learning classifiers. Large-scale evaluation on two real-world datasets has demonstrated their attack's effectiveness. Moreover, the authors show their framework can easily incorporate different defense mechanisms, such as differential privacy, to allow a comprehensive evaluation of membership inference risks.
Membership Inference Against Machine Learning. Shokri et al. [38] present the first membership inference attack against machine learning models. The key contribution of this work is the proposal of shadow model training, which aims at mimicking the target model's behavior to generate training data for the attack model.

The first adversary in the current paper follows a very similar setting. We have shown that one shadow model and one attack model are sufficient to achieve an effective attack compared to the proposal of multiple shadow models and attack models by Shokri et al. [38]. Moreover, we show that data transferring attack can bypass the expensive synthetic data generation scheme and achieve a very similar performance. Another major contribution of our paper is the two effective defense mechanisms, such as dropout and model stacking. Many recent works have studied membership inference against machine learning as well from different angles [25], [17], [48], [26].

Attacks Against Machine Learning. Besides membership inference, there exist multiple other types of attacks against ML models. Fredrikson et al. [13] present the model inversion attack in biomedical data setting. In this scenario, an attacker aims to infer the missing attributes of her victim, relying on the output of a trained ML model. Later, model inversion attack is generalized to a broader scenario [12]. For instance, the authors show that it is feasible for an attacker to reconstruct a recognizable face of her victim with model inversion.

Tramèr et al. [43] propose another attack on ML models, namely model extraction attack. This attack aims at stealing the ML model, i.e., the model's learned parameters, through the output of MLaaS API itself. They first propose an equationsolving attack, where an attacker queries MLaaS API multiple times and use the output posteriors to construct a set of equations. By solving these equations, the attacker can obtain the weight of the ML model. Tramèr et al. [43] further propose a path-finding algorithm, which is the first practical method to steal decision trees. In the end, Tramèr et al. show that even ML models which do not provide prediction posteriors but only prediction class labels can still be stolen with retraining strategies, such as active learning. It is worth noting that due to the effectiveness of the model extraction attack, we do not consider hiding posteriors as one valid defense mechanism in this paper.

Another major family of attacks against machine learning is adversarial examples [33], [44], [8], [23], [42], [34], [46]. In this setting, an attacker adds a controlled amount of noise to a data point which aims to fool a trained ML model to misclassify the data point. Adversarial examples can cause severe risks in multiple domains, such as autonomous driving, and voice recognition. On the other hand, researchers have recently shown that adversarial examples can also help to protect users' privacy in online social networks [31], [22], [49].

Privacy-Preserving Machine Learning. Another relevant line of work is privacy-preserving machine learning [24], [19], [11], [10], [14], [28], [5], [6], [1]. Mohassel and Zhang [28] present efficient protocols for training linear regression, logistic regression, and neural networks in a privacy-preserving manner. Their protocols fall in the two-server mode where data is distributed over two non-colluding servers. The authors use 
two-party computation to implement these protocols. Bonawitz et al. [5] propose a protocol for secure aggregation over highdimensional data, which is a key component for distributed machine learning. The protocol is also based on multi-party computation, and the authors show its security under both honest-but-curious and active adversary setting. Large-scale evaluation demonstrates the efficiency of this protocol.

Besides privacy-preserving model training, other works study privacy-preserving classification. Bost et al. [6] design three protocols based on homomorphic encryption. They concentrate on three ML classifiers including hyperplane decision, Naive Bayes, and decision trees, and show that their protocols can be efficiently executed. Based on the scheme of Bost et al., Backes et al. [1] build a privacy-preserving random forests classifier for medical diagnosis. Besides the above, many recent works have tackled security and privacy in machine learning from various perspectives [39], [16], [9], [37], [7], [27], [20], [45], [36], [21], [40], [29], [41], [20].

\section{CONCLUSION}

Training data is a key factor that drives machine learning model being widely adopted in real-world applications. However, ML models suffer from membership privacy risks. The existing membership inference attacks have shown effective performance, but their applicability is limited due to strong assumptions on the threat model. In this paper, we gradually relax these assumptions towards a more broadly applicable attack scenario.

Our first adversary utilizes only one shadow model. Extensive experiments show that this attack achieves a very similar performance as the previous one which utilizes multiple shadow models. As shadow models are established through MLaaS, our proposal notably reduces the cost of conducting the attack. We further perform the combining attack which does not require knowledge of the type of classifiers used in the target model.

The attack assumption is further relaxed for the second adversary, who does not have access to a dataset that comes from the same distribution as the training data of the target model. This is a more realistic attack scenario, but the previously proposed synthetic data generation solution can only be applied in specific cases. In contrast, we propose data transferring attacks, where the adversary utilizes another dataset to build a shadow model and generates the corresponding data to attack the target model. Through experiments, we have discovered that data transferring attack also achieves strong membership inference while being more general, realistic and widely applicable.

The third adversary has a minimal set of assumptions, i.e., she does not need to construct any shadow model and her attack is performed in an unsupervised way. We show that even in such a simple setting, membership inference is still effective.

Our evaluation is comprehensive and fully demonstrates the severe threat of membership privacy in ML models under those generalized conditions on 8 diverse datasets.

To remedy the situation, we propose two defense mechanisms. As we show the connection between overfitting and sensitivity to membership inference attacks, we investigate techniques that are designed to reduce overfitting. The first one, namely dropout, randomly deletes a certain proportion of edges in each training iteration in a fully connected neural network, while the second approach, namely model stacking, organizes multiple ML models in a hierarchical way. Extensive evaluation shows that indeed our defense techniques are able to largely reduce membership inference attack's performance, while maintaining a high-level utility, i.e., high target model's prediction accuracy.

\section{ACKNOWLEDGMENTS}

The research leading to these results has received funding from the European Research Council under the European Union's Seventh Framework Programme (FP7/2007-2013)/ ERC grant agreement no. 610150-imPACT.

\section{REFERENCES}

[1] M. Backes, P. Berrang, M. Bieg, R. Eils, C. Herrmann, M. Humbert, and I. Lehmann, "Identifying Personal DNA Methylation Profiles by Genotype Inference," in Proceedings of the 2017 IEEE Symposium on Security and Privacy (S\&P). IEEE, 2017, pp. 957-976. 13, 14

[2] M. Backes, P. Berrang, M. Humbert, and P. Manoharan, "Membership Privacy in MicroRNA-based Studies," in Proceedings of the 2016 ACM SIGSAC Conference on Computer and Communications Security (CCS). ACM, 2016, pp. 319-330. 1, 13

[3] M. Backes, M. Humbert, J. Pang, and Y. Zhang, "walk2friends: Inferring Social Links from Mobility Profiles," in Proceedings of the 2017 ACM SIGSAC Conference on Computer and Communications Security (CCS). ACM, 2017, pp. 1943-1957. 9

[4] P. Berrang, M. Humbert, Y. Zhang, I. Lehmann, R. Eils, and M. Backes, "Dissecting Privacy Risks in Biomedical Data," in Proceedings of the 2018 IEEE European Symposium on Security and Privacy (Euro S\&P). IEEE, 2018. 8

[5] K. Bonawitz, V. Ivanov, B. Kreuter, A. Marcedone, H. B. McMahan, S. Patel, D. Ramage, A. Segal, and K. Seth, "Practical Secure Aggregation for Privacy-Preserving Machine Learning," in Proceedings of the 2017 ACM SIGSAC Conference on Computer and Communications Security (CCS). ACM, 2017, pp. 1175-1191. 13, 14

[6] R. Bost, R. A. Popa, S. Tu, and S. Goldwasser, "Machine Learning Classification over Encrypted Data," in Proceedings of the 2015 Network and Distributed System Security Symposium (NDSS). Internet Society, 2015. 13, 14

[7] X. Cao and N. Z. Gong, "Mitigating Evasion Attacks to Deep Neural Networks via Region-based Classification," in Proceedings of the 2017 Annual Computer Security Applications Conference (ACSAC). ACM, 2017, pp. 278-287. 14

[8] N. Carlini and D. Wagner, "Towards Evaluating the Robustness of Neural Networks," in Proceedings of the 2017 IEEE Symposium on Security and Privacy (S\&P). IEEE, 2017, pp. 39-57. 13

[9] Q. Chen, C. Xiang, M. Xue, B. Li, N. Borisov, D. Kaarfar, and H. Zhu, "Differentially Private Data Generative Models," CoRR abs/1812.02274, 2018. 14

[10] N. Dowlin, R. Gilad-Bachrach, K. Laine, K. Lauter, M. Naehrig, and J. Wernsing, "CryptoNets: Applying Neural Networks to Encrypted Data with High Throughput and Accuracy," in Proceedings of the 2016 International Conference on Machine Learning (ICML). JMLR, 2016, pp. 201-210. 13

[11] Z. Erkin, M. Franz, J. Guajardo, S. Katzenbeisser, I. Lagendijk, and T. Toft, "Privacy-Preserving Face Recognition," Symposium on Privacy Enhancing Technologies Symposium, 2009. 13

[12] M. Fredrikson, S. Jha, and T. Ristenpart, "Model Inversion Attacks that Exploit Confidence Information and Basic Countermeasures," in Proceedings of the 2015 ACM SIGSAC Conference on Computer and Communications Security (CCS). ACM, 2015, pp. 1322-1333. 1, 13 
[13] M. Fredrikson, E. Lantz, S. Jha, S. Lin, D. Page, and T. Ristenpart, "Privacy in Pharmacogenetics: An End-to-End Case Study of Personalized Warfarin Dosing," in Proceedings of the 2014 USENIX Security Symposium (USENIX Security). USENIX, 2014, pp. 17-32. 9, 13

[14] A. Gascón, P. Schoppmann, B. Balle, M. Raykova, J. Doerner, S. Zahur, and D. Evans, "Privacy-Preserving Distributed Linear Regression on High-Dimensional Data," Symposium on Privacy Enhancing Technologies Symposium, 2017. 13

[15] I. Goodfellow, J. Shlens, and C. Szegedy, "Explaining and Harnessing Adversarial Examples," in Proceedings of the 2015 International Conference on Learning Representations (ICLR), 2015. 1

[16] L. Hanzlik, Y. Zhang, K. Grosse, A. Salem, M. Augustin, M. Backes, and M. Fritz, "MLCapsule: Guarded Offline Deployment of Machine Learning as a Service," CoRR abs/1808.00590, 2018. 14

[17] J. Hayes, L. Melis, G. Danezis, and E. D. Cristofaro, "LOGAN: Evaluating Privacy Leakage of Generative Models Using Generative Adversarial Networks," CoRR abs/1705.07663, 2017. 13

[18] N. Homer, S. Szelinger, M. Redman, D. Duggan, W. Tembe, J. Muehling, J. V. Pearson, D. A. Stephan, S. F. Nelson, and D. W. Craig, "Resolving Individuals Contributing Trace Amounts of DNA to Highly Complex Mixtures Using High-Density SNP Genotyping Microarrays," PLOS Genetics, 2008. 13

[19] Y. Huang, L. Malka, D. Evans, and J. Katz, "Efficient PrivacyPreserving Biometric Identification," in Proceedings of the 2011 Network and Distributed System Security Symposium (NDSS). Internet Society, 2011. 13

[20] T. Hunt, C. Song, R. Shokri, V. Shmatikov, and E. Witchel, "Chiron: Privacy-preserving Machine Learning as a Service," CoRR $a b s / 1803.05961,2018.14$

[21] M. Jagielski, A. Oprea, B. Biggio, C. Liu, C. Nita-Rotaru, and B. Li, "Manipulating Machine Learning: Poisoning Attacks and Countermeasures for Regression Learning," in Proceedings of the 2018 IEEE Symposium on Security and Privacy $(S \& P)$. IEEE, 2018. 14

[22] J. Jia and N. Z. Gong, "AttriGuard: A Practical Defense Against Attribute Inference Attacks via Adversarial Machine Learning," in Proceedings of the 2018 USENIX Security Symposium (USENIX Security). USENIX, 2018. 13

[23] B. Li and Y. Vorobeychik, "Scalable Optimization of Randomized Operational Decisions in Adversarial Classification Settings," in Proceedings of the 2015 International Conference on Artificial Intelligence and Statistics (AISTATS). PMLR, 2015, pp. 599-607. 13

[24] J. Liu, M. Juuti, Y. Lu, and N. Asokan, "Oblivious Neural Network Predictions via MiniONN Transformations," in Proceedings of the 2017 ACM SIGSAC Conference on Computer and Communications Security (CCS). ACM, 2017, pp. 619-631. 13

[25] Y. Long, V. Bindschaedler, and C. A. Gunter, "Towards Measuring Membership Privacy," CoRR abs/1712.09136, 2017. 13

[26] Y. Long, V. Bindschaedler, L. Wang, D. Bu, X. Wang, H. Tang, C. A. Gunter, and K. Chen, "Understanding Membership Inferences on WellGeneralized Learning Models," CoRR abs/1802.04889, 2018. 13

[27] L. Melis, C. Song, E. D. Cristofaro, and V. Shmatikov, "Inference Attacks Against Collaborative Learning," CoRR abs/1805.04049, 2018. 14

[28] P. Mohassel and Y. Zhang, "SecureML: A System for Scalable PrivacyPreserving Machine Learning," in Proceedings of the 2017 IEEE Symposium on Security and Privacy (S\&P). IEEE, 2017, pp. 19-38. 13

[29] M. Nasr, R. Shokri, and A. Houmansadr, "Machine Learning with Membership Privacy using Adversarial Regularization," in Proceedings of the 2018 ACM SIGSAC Conference on Computer and Communications Security (CCS). ACM, 2018. 14

[30] S. J. Oh, M. Augustin, B. Schiele, and M. Fritz, "Towards ReverseEngineering Black-Box Neural Networks," in Proceedings of the 2018 International Conference on Learning Representations (ICLR), 2018. 1, 4

[31] S. J. Oh, M. Fritz, and B. Schiele, "Adversarial Image Perturbation for Privacy Protection - A Game Theory Perspective," in Proceedings of the 2017 IEEE International Conference on Computer Vision (ICCV). IEEE, 2017, pp. 1482-1491. 13
[32] J. Pang and Y. Zhang, "DeepCity: A Feature Learning Framework for Mining Location Check-Ins," in Proceedings of the 2017 International Conference on Weblogs and Social Media (ICWSM). AAAI, 2017, pp. 652-655. 9

[33] N. Papernot, P. D. McDaniel, I. Goodfellow, S. Jha, Z. B. Celik, and A. Swami, "Practical Black-Box Attacks Against Machine Learning," in Proceedings of the 2017 ACM Asia Conference on Computer and Communications Security (ASIACCS). ACM, 2017, pp. 506-519. 13

[34] N. Papernot, P. D. McDaniel, S. Jha, M. Fredrikson, Z. B. Celik, and A. Swami, "The Limitations of Deep Learning in Adversarial Settings," in Proceedings of the 2016 IEEE European Symposium on Security and Privacy (Euro S\&P). IEEE, 2016, pp. 372-387. 13

[35] A. Pyrgelis, C. Troncoso, and E. D. Cristofaro, "Knock Knock, Who's There? Membership Inference on Aggregate Location Data," in Proceedings of the 2018 Network and Distributed System Security Symposium (NDSS). Internet Society, 2018. 1, 9, 13

[36] B. D. Rouhani, H. Chen, and F. Koushanfar, "DeepSigns: A Generic Watermarking Framework for IP Protection of Deep Learning Models," CoRR abs/1804.00750, 2018. 14

[37] B. D. Rouhani, M. S. Riazi, and F. Koushanfar, "DeepSecure: Scalable Provably-Secure Deep Learning," CoRR abs/1705.08963, 2017. 14

[38] R. Shokri, M. Stronati, C. Song, and V. Shmatikov, "Membership Inference Attacks Against Machine Learning Models," in Proceedings of the 2017 IEEE Symposium on Security and Privacy (S\&P). IEEE, 2017, pp. 3-18. 1, 2, 3, 4, 6, 7, 10, 11, 13

[39] C. Song, T. Ristenpart, and V. Shmatikov, "Machine Learning Models that Remember Too Much," in Proceedings of the 2017 ACM SIGSAC Conference on Computer and Communications Security (CCS). ACM, 2017, pp. 587-601. 14

[40] O. Suciu, R. Mărginean, Y. Kaya, H. D. III, and T. Dumitraş, "When Does Machine Learning FAIL? Generalized Transferability for Evasion and Poisoning Attacks," CoRR abs/1803.06975, 2018. 14

[41] F. Tramer and D. Boneh, "Slalom: Fast, Verifiable and Private Execution of Neural Networks in Trusted Hardware," CoRR abs/1806.03287, 2018. 14

[42] F. Tramèr, A. Kurakin, N. Papernot, I. Goodfellow, D. Boneh, and P. McDaniel, "Ensemble Adversarial Training: Attacks and Defenses," in Proceedings of the 2017 International Conference on Learning Representations (ICLR), 2017. 13

[43] F. Tramér, F. Zhang, A. Juels, M. K. Reiter, and T. Ristenpart, "Stealing Machine Learning Models via Prediction APIs," in Proceedings of the 2016 USENIX Security Symposium (USENIX Security). USENIX, 2016, pp. 601-618. 1, 4, 13

[44] Y. Vorobeychik and B. Li, "Optimal Randomized Classification in Adversarial Settings," in Proceedings of the 2014 International Conference on Autonomous Agents and Multi-agent Systems (AAMAS), 2014, pp. 485-492. 13

[45] B. Wang and N. Z. Gong, "Stealing Hyperparameters in Machine Learning," in Proceedings of the 2018 IEEE Symposium on Security and Privacy $(S \& P)$. IEEE, 2018. 1, 4, 14

[46] W. Xu, D. Evans, and Y. Qi, "Feature Squeezing: Detecting Adversarial Examples in Deep Neural Networks," in Proceedings of the 2018 Network and Distributed System Security Symposium (NDSS). Internet Society, 2018. 13

[47] D. Yang, D. Zhang, and B. Qu, "Participatory Cultural Mapping Based on Collective Behavior Data in Location-Based Social Networks," ACM Transactions on Intelligent Systems and Technology, 2016. 3

[48] S. Yeom, I. Giacomelli, M. Fredrikson, and S. Jha, "Privacy Risk in Machine Learning: Analyzing the Connection to Overfitting," in Proceedings of the 2018 IEEE Computer Security Foundations Symposium $(C S F)$. IEEE, 2018. 4, 8, 13

[49] Y. Zhang, M. Humbert, T. Rahman, C.-T. Li, J. Pang, and M. Backes, "Tagvisor: A Privacy Advisor for Sharing Hashtags," in Proceedings of the 2018 Web Conference (WWW). ACM, 2018, pp. 287-296. 9, 13

[50] Y. Zhang, M. Humbert, B. Surma, P. Manoharan, J. Vreeken, and M. Backes, "CTRL+Z: Recovering Anonymized Social Graphs," CoRR abs/1711.05441, 2017. 9 